\title{
TEORÍA DE CONJUNTOS APLICADA A POBLACIONES DE LEUCOCITOS, LINFOCITOS Y CD4 DE PACIENTES CON VIH. Predicción de linfocitos T CD4, de aplicación clínica
}

\author{
Javier Rodríguez ${ }^{1}$, Signed Prieto ${ }^{2}$, Pedro BernaL ${ }^{3}$, Carlos Pérez ${ }^{4}$, Catalina Correa ${ }^{5}$, Sarith Viterr ${ }^{6}$ \\ Grupo INSIGHT - UnIVERSIDAD MILITAR NUEVA GRANADA \\ ${ }^{1}$ MD. Director Grupo Insight-Centro de Investigaciones Clínica del Country. Director de la Linea de Profundización \\ e internado especial "Física y Matemáticas aplicadas a la Medicina". Universidad Militar Nueva Granada. \\ ${ }^{2}$ Investigadora Grupo Insight - Centro de Investigaciones Clínica del Country. \\ ${ }^{3}$ Investigador Grupo Insight - Centro de Investigaciones Clínica del Country. Estudiante de Ingeniería \\ de Sistemas Universidad Nacional de Colombia. \\ ${ }^{4} \mathrm{MD}$. Infectólogo. Docente Facultad de Medicina. Universidad Militar Nueva Granada. \\ ${ }^{5}$ Psicóloga. Investigadora Grupo Insight - Centro de Investigaciones Clínica del Country. Docente de la Línea de \\ Profundizacióne Internado Especial "Físicay Matemáticasaplicadas alaMedicina". Universidad Militar Nueva Granada. \\ "Interna especial de la Línea de Profundización e Internado Especial "Física y Matemáticas aplicadas a \\ la Medicina". Universidad Militar Nueva Granada - Centro de Investigaciones Clínica del Country.
}

\begin{abstract}
Resumen
Estudios en poblaciones de leucocitos y linfocitos del sistema inmune evidencian la pérdida de linfocitos CD4 a través del tiempo en el desarrollo de SIDA, lo cual tiene implicaciones en el inicio y seguimiento del tratamiento, así como en la predicción de mortalidad. Se han desarrollado algunas predicciones sobre la variabilidad y el conteo de $\mathrm{CD} 4$. El objetivo de este trabajo es predecir las poblaciones de CD4 con base en la información de leucocitos y linfocitos del cuadro hemático, mediante la teoría de conjuntos. Para ello, a partir de 7 triplas de datos de la cantidad de leucocitos $/ \mathrm{ml}^{3}$, linfocitos $/ \mathrm{ml}^{3}$ y la subpoblación de $\mathrm{CD} 4 / \mu \mathrm{L}$ mediante citometría de flujo de pacientes específicos, se desarrolló una inducción que generó cuatro conjuntos A, B, C y D. Basándose en éstos se cuantificaron 103 cuadros hemáticos, estableciendo su pertenencia a cada conjunto de acuerdo con las diferentes distribuciones de las tres poblaciones respecto a rangos de 1.000 leucocitos $/ \mathrm{ml}^{3}$. Posteriormente se evaluó $A \cup C, B \cup D$ y la intersección entre las dos uniones. Finalmente se estableció el número de elementos componentes de estos conjuntos, así como el porcentaje de pertenencia respecto a la totalidad de casos de cada rango.
\end{abstract}

Se estableció un porcentaje de acierto superior al $80 \%$ para 5 de los 8 rangos medidos, y que los rangos de leucocitos inferiores a 5.000 y $4.000 / \mathrm{ml}^{3}$ se pueden asociar a menos de $570 \mathrm{CD} 4 / \mu \mathrm{L}$ con un porcentaje de efectividad de $90 \%$ y $100 \%$ respectivamente, de tal modo que a medida que disminuye la cantidad de leucocitos desde el rango de 6.000 el porcentaje de aciertos de la predicción entre las tres medidas es más efectiva. Se concluye que la teoría de conjuntos aplicada a las poblaciones de leucocitos, linfocitos, y $\mathrm{CD} 4$ revela una autoorganización matemática objetiva, reproducible y de aplicación clínica para la predicción de rangos de $\mathrm{CD} 4 / \mu \mathrm{l}$, cuyo uso puede reducir recursos y costos.

Palabras clave: Cuadro Hemático, CD4, VIH, SIDA, Teoría de conjuntos.

* Correspondencia: grupoinsiht2025@yahoo.es

Recibido: julio 26 de 2011 Aceptado: octubre 20 de 2011 


\title{
SET THEORY APPLIED TO LEUKOCYTES, LYMPHOCYTES AND CD4 IN POPULATIONS OF HIV PATIENTS Prediction of T-CD4 lymphocytes, for clinical application
}

\begin{abstract}
Summary
Studies on the immune system leukocytes and lymphocytes populations evidence the loss of CD4 lymphocytes over time in the development of has implications on the treatment start and follow-up as well as on the mortality prediction. A number of predictions have been developed regarding the $\mathrm{CD} 4$ variability and count. The aim of this paper is to predict the CD4 populations based on the information about the leukocytes and lymphocytes in the complete blood count through the set theory. With this purpose, starting from 7 data triplets of the amount of leukocytes $/ \mathrm{ml}^{3}$, lymphocytes/ $\mathrm{ml}^{3}$ and the $\mathrm{CD} 4 / \mu \mathrm{L}$ sub-population through flux cytometry in specific patients, an induction that generated four A, B, C and D sets was developed. Based on those sets, 103 complete blood counts were quantified, by setting their belonging to each set according to the different distributions of the three populations with respect to ranges of 1000 leukocytes $/ \mathrm{ml}^{3}$. Subsequently, A $\cup C, B \cup D$ and the intersection between the two bonds were assessed. Finally, the number of elements composing these sets was determined as well as the belonging percentage in reference to the totality of cases in each range.

A percentage of higher than $80 \%$ success for 5 of the 8 measured ranges became established, and the fact that the leukocytes ranges below 5000 a $4000 / \mathrm{ml}^{3}$ can be associated to less than $570 \mathrm{CD} 4 /$ $\mu \mathrm{L}$, with $90 \%$ and $100 \%$ effectiveness percentage, respectively, so that, as the amount of leukocytes decreases from the range of 6000 , the percentage of correct forecasts between the three measures is more effective. In conclusion, the set theory applied to the leukocytes, lymphocytes, and CD4 populations reveals an objective mathematical auto-organization, which is reproducible and has clinical application for predicting $\mathrm{CD} 4 / \mathrm{\mu l}$ ranges, whose use can reduce resources and costs.
\end{abstract}

Key words: Complete blood count, CD4, HIV, AIDS, Set theory.

\section{TEORIA DE CONJUNTOS APLICADA A POPULAÇÕES DE LEUCÓCITOS, LINFÓCITOS Y CD4 DE PACIENTES COM HIV. Predição de linfócitos T CD4, de aplicação clínica}

\begin{abstract}
Resumo
Estudos em populações de leucócitos e linfócitos do sistema imunológico evidenciam a perda de linfócitos CD4 através do tempo no desenvolvimento da AIDS, que tem implicações no inicio e acompanhamento do tratamento, bem como na predição de mortalidade. Foram desenvolvidas algumas predições sobre a variabilidade e a contagem de CD4. O objetivo deste trabalho é predizer as populações de $\mathrm{CD} 4$ com base na informação de leucócitos e linfócitos do quadro hemático, mediante a teoría de conjuntos. Para isso, a partir de 7 triplas de dados da quantidade de leucócitos/ $\mathrm{ml}^{3}$, linfócitos $/ \mathrm{ml}^{3}$ e a subpopulação de $\mathrm{CD} 4 / \mu \mathrm{L}$ mediante citometria de fluxo de pacientes específicos, desenvolveu-se uma indução que gerou quatro conjuntos A, B, C e D. Baseando-se nestes quantificaram-se 103 quadros hemáticos, estabelecendo sua atribuição a cada conjunto de acordo com as diferentes distribuições das três populações com respeito a intervalos de 1.000 leucócitos $/ \mathrm{ml}^{3}$. Posteriormente avaliou-se $A \cup C, B \cup D$ e a intersecção entre as duas uniões. Finalmente estabeleceuse o número de elementos componentes destes conjuntos, bem como a porcentagem de atribuição com respeito à totalidade de casos de cada intervalo.
\end{abstract}




\begin{abstract}
Estabeleceu-se uma porcentagem de acerto superior a $80 \%$ para 5 dos 8 intervalos medidos, $e$ que os intervalos de leucócitos inferiores a 5.000 e $4.000 / \mathrm{ml}^{3}$ podem ser associados a menos de $570 \mathrm{CD} 4 / \mu \mathrm{L}$ com uma porcentagem de efetividade de $90 \%$ e $100 \%$ respectivamente, de tal modo que a medida que diminui a quantidade de leucócitos desde o intervalo de 6.000 a porcentagem de acertos da predição entre as três medidas é mais efetiva. Conclui-se que a teoría de conjuntos aplicada às populações de leucócitos, linfócitos, e CD4 revela uma auto-organização matemática objetiva, reproduzivel e de aplicação clínica para a predição de intervalos de $\mathrm{CD} 4 / \mu \mathrm{l}$, cujo uso pode reduzir recursos e custos.
\end{abstract}

Palavras chave: Quadro Hemático, CD4, HIV, AIDS, Teoria dos conjuntos.

\section{Introducción}

La pandemia de la infección por el VIH presenta un total de 33.3 millones de personas con esta enfermedad a nivel mundial (año 2.009), una incidencia de 2.6 millones de casos y 1.8 millones de muertes por SIDA para el mismo año (1). En 2.007, África Subsahariana fue catalogada como la región más afectada por el VIH, 67\% de sus habitantes portaban el virus y representó el 72\% de las muertes por SIDA a nivel mundial. En 2.008 en Latinoamérica la cifra estimada de nuevas infecciones por este virus fue de 170.000, con una prevalencia regional de $0.6 \%$ (epidemia de bajo nivel y concentrada), donde el grupo de hombres que tienen sexo con hombres es el de más alto riesgo, con una probabilidad de contraer el $\mathrm{VIH}$ tres veces más alta con respecto a otros grupos poblacionales. En Colombia la prevalencia de VIH en la población de 15 a 49 años se estima en $0.7 \%$, con un total de casos, desde 1983 hasta 2009, de 71.653 (2); y hasta la semana 49 de 2.010, sólo Bogotá reportó al grupo de ITS del Sivigila un total de 1.267 casos (3).

El retrovirus VIH afecta básicamente a las células T y los macrófagos que expresan el CD4, además de los ganglios linfáticos y las células dendríticas foliculares (4). Aunque el conteo de linfocitos es determinado mediante el cuadro hemático, que es un examen de gran cobertura y bajo costo, para el establecimiento del nivel de la subpoblación de linfocitos CD4 es necesaria la citometría de flujo; sobre la cual, trabajos con implementación de citómetros de flujo simplificados cargables por batería o paneles solares han buscado disminuir su costo (5). Sin embargo su cobertura es muy baja en países en vías de desarrollo, dado que requieren reactivos, así como personal altamente calificado.
Se ha observado relación entre el número absoluto y el porcentaje de CD4 en los pacientes con infección $\mathrm{VIH} / \mathrm{SIDA}$, donde los que tienen menos del 14\% de CD4 tienen menos de $200 \mathrm{CD} 4 / \mu \mathrm{l}^{3}$; entre el 14$28 \%$ tienen entre 200 y 500 y los que tienen más del 28\% tienen más de 500 linfocitos $\mathrm{CD} 4 / \mu \mathrm{l}^{3}$ (6). Se ha encontrado que generalmente los pacientes que presentan menos de 1.500 linfocitos totales tienen menos de 500 linfocitos $\mathrm{CD} 4 / \mathrm{\mu l}^{3}$, mientras que quienes tienen más de 1.500 linfocitos totales normalmente tienen más de 200 linfocitos CD4/ $\mathrm{ll}^{3}$ (6). Sin embargo, debido a la complejidad y variabilidad del sistema no se ha podido establecer una predicción del comportamiento de estas poblaciones para cada paciente.

La aplicación de teorías físicas y matemáticas en diferentes áreas de la medicina ha permitido establecer órdenes de aplicación experimental, clínica y epidemiológica. En el campo de la inmunología se desarrolló una teoría para la predicción del fenómeno de unión de péptidos al HLA clase II, con base en la probabilidad, combinatoria y entropía, que predijo correctamente el estado de unión o no unión de la totalidad de péptidos estudiados, tanto teóricos, como sintéticos y naturales (7). También se realizó una caracterización matemática del comportamiento del repertorio T específico contra el alérgeno Poa p9, así como del patrón Th2 con base en la ley de Zipf-Mandelbrot, estableciendo diferencias en las dimensiones fractales en presencia y ausencia de interferón $\alpha$ en la muestra, evidenciando que la dimensión fractal estadística es apropiada para caracterizar la fisiología del sistema inmune y puede tener implicaciones generales para el desarrollo de la inmunología (8).

La teoría de conjuntos es una teoría matemática fundamentada en nociones como la de pertenencia, 
así como el cumplimiento de una serie de axiomas que definen las relaciones matemáticas entre los elementos. Esta teoría permite la diferenciación de elementos particulares con base en conjuntos generales mediante la realización de operaciones como la unión, la intersección, la diferencia y la diferencia simétrica.

Dada la magnitud de la pandemia del VIH/SIDA a nivel mundial, especialmente en zonas de bajos recursos económicos, resulta de vital importancia establecer metodologías que predigan el comportamiento de estas poblaciones celulares con base en la información obtenida desde el cuadro hemático. El propósito de este trabajo es establecer una caracterización matemática del comportamiento de leucocitos, linfocitos totales y linfocitos T CD4, con base en la teoría de conjuntos, que permita la predicción de linfocitos $\mathrm{T}$ CD4 con base en la información del cuadro hemático.

\section{Metodología}

\section{Definiciones}

Conjuntos definidos para la evaluación de las poblaciones de leucocitos $/ \mathrm{ml}^{3}$, linfocitos $/ \mathrm{ml}^{3}$ y $\mathrm{CD} 4 / \mu \mathrm{l}^{3}$ :
A: $\{(x, y, z) / x \geq 6.800 \wedge y \geq 1.800\}$
B: $\{(x, y, z) / x \geq 6.800 \Lambda z \geq 300\}$
C: $\{(x, y, z) / x<6.800 \wedge y \leq 2.660\}$
D: $\{(x, y, z) / x<6.800 \wedge z \leq 570\}$

Donde $(\boldsymbol{x}, \boldsymbol{y}, \boldsymbol{z})$ se define como una tripla de valores donde $\boldsymbol{x}$ representa el número de leucocitos, $\boldsymbol{y}$, el número de linfocitos y $\mathbf{z}$ el conteo de linfocitos T CD4.

\section{Procedimiento}

Se desarrolló una inducción a partir de 7 pacientes específicos escogidos al observar las distribuciones clínicas de leucocitos $/ \mathrm{ml}^{3}$, linfocitos $/ \mathrm{ml}^{3}$ y CD4/ $/ \mathrm{l}^{3}$ de 110 casos. Estos casos fueron escogidos por presentar características representativas de la totalidad de posibles distribuciones, de tal modo que permiten establecer un orden matemático de la totalidad del fenómeno, ver tabla 1. Para lograr la simplificación matemática, en este estudio se excluyeron la carga viral, hemoglobina, edad, sexo o medicamentos utilizados por los pacientes. De acuerdo con las observaciones realizadas en los casos escogidos para la realización de la inducción, se establecieron características de las poblaciones de leucocitos $/ \mathrm{ml}^{3}$ respecto a linfocitos/ $\mathrm{ml}^{3}$ y respecto a CD4/ $\mathrm{ul}^{3}$ que fueron representadas con base en la teoría de conjuntos, para mediante el establecimiento de la pertenencia o no pertenencia de cada registro a los 4 conjuntos establecidos poder encontrar un orden matemático de los conteos.

Para establecer el comportamiento de la presentación de leucocitos $/ \mathrm{ml}^{3}$ respecto a linfocitos $/ \mathrm{ml}^{3}$ y CD4/ $\mathrm{\mu l}^{3}$, se evaluó la unión entre los conjuntos A y C, y entre los conjuntos $\mathrm{B}$ y $\mathrm{D}$, para posteriormente establecer la intersección entre $A \cup C$ y $B \cup D$.

Posteriormente se contaron registros de leucocitos/ $\mathrm{ml}^{3}$, linfocitos $/ \mathrm{ml}^{3}$ y recuentos de la subpoblación de $\mathrm{CD} 4 / \mathrm{\mu l}^{3}$, medidos con citometría de flujo, de 103 pacientes, provenientes del archivo personal del Infectólogo que realiza esta investigación.

Los registros fueron ordenados de mayor a menor de acuerdo con el número de leucocitos estableciendo rangos de a 1.000 leucocitos $/ \mathrm{ml}^{3}$, en tanto que valores mayores a $10.000 / \mathrm{ml}^{3}$ corresponden a un solo rango, y valores menores a $4.000 / \mathrm{ml}^{3}$ constituyen otro rango, obteniendo 8 rangos en total. Estos rangos fueron establecidos para poder observar relaciones matemáticas entre las poblaciones independientemente del tiempo y de la evolución del paciente.

De acuerdo con la inducción realizada, los 103 registros restantes fueron evaluados, estableciendo su pertenencia o no pertenencia a los conjuntos $A \cup C$ y $\mathrm{B} \cup \mathrm{D}$, así como al conjunto $(\mathrm{A} \cup \mathrm{C}) \cap(\mathrm{B} \cup \mathrm{D})$.

Los resultados fueron evaluados para los 8 rangos de leucocitos, determinando el número de elementos que hacen parte de cada conjunto en cada rango y el porcentaje al que corresponden de acuerdo al número total hallado para cada rango. Adicionalmente se establecieron estos mismos valores para la totalidad de la muestra.

Con base en los resultados obtenidos se desarrolló un software en lenguaje $\mathrm{C}++$ basado en el álgebra de conjuntos, que permite predecir el rango de CD4 a partir del número de leucocitos y de linfocitos del cuadro hemático para cada caso particular, con el objetivo de encontrar órdenes matemáticos subyacentes y disminuir costos y recursos en la obtención del valor de CD4 con citometría de flujo. 
Tabla 1. Valores de leucocitos $/ \mathrm{ml}^{3}$, linfocitos $/ \mathrm{ml}^{3}$ y CD4/ $/ \mathrm{l}^{3}$ para los 110 casos evaluados. Los 7 casos correspondientes a la inducción se encuentran resaltados en gris. Los valores en negrilla son aquellos que no corresponden a alguno de los conjuntos establecidos.

\begin{tabular}{|c|c|c|c|c|}
\hline No. & $\begin{array}{l}\text { Clasificación } \\
\text { actual según } \\
\text { CDC }\end{array}$ & $\begin{array}{l}\text { Leucocitos/ } \\
\mathrm{ml}^{3}\end{array}$ & $\begin{array}{c}\text { Linfocitos/ } \\
\mathrm{ml}^{3}\end{array}$ & $\begin{array}{c}\text { Conteo de } \\
\mathrm{CD} 4 / \mu \mathrm{l}^{3}\end{array}$ \\
\hline 1 & $\mathrm{~A} 1$ & 13.180 & 2.160 & 362 \\
\hline 2 & A1 & 11.400 & 1.950 & 728 \\
\hline 3 & $\mathrm{~A} 1$ & 11.030 & 3.560 & 1.039 \\
\hline 4 & A2 & 10.480 & 2.830 & 681 \\
\hline 5 & A2 & 10.110 & 2.620 & 294 \\
\hline 6 & $\mathrm{~A} 1$ & 10.000 & 2.480 & 509 \\
\hline 7 & $\mathrm{~A} 2$ & 9.820 & 3.150 & 482 \\
\hline 8 & $\mathrm{C} 2$ & 9.800 & 1.300 & 396 \\
\hline 9 & A3 & 9.560 & 1.900 & 100 \\
\hline 10 & A1 & 9.440 & 3.080 & 506 \\
\hline 11 & $\mathrm{~A} 2$ & 9.200 & 2.971 & 458 \\
\hline 12 & $\mathrm{~A} 1$ & 8.990 & 2.520 & 187 \\
\hline 13 & B1 & 8.920 & 3.870 & 694 \\
\hline 14 & A1 & 8.800 & 2.974 & 865 \\
\hline 15 & $\mathrm{~B} 2$ & 8.780 & 2.680 & 443 \\
\hline 16 & $\mathrm{~A} 1$ & 8.500 & 2.218 & 639 \\
\hline 17 & $\mathrm{~A} 1$ & 8.180 & 2.740 & 511 \\
\hline 18 & A3 & 7.900 & 2.733 & 46 \\
\hline 19 & B3 & 7.900 & 1.610 & 24 \\
\hline 20 & A2 & 7.800 & 2.043 & 531 \\
\hline 21 & A1 & 7.800 & 4.000 & 703 \\
\hline 22 & A1 & 7.700 & 3.141 & 494 \\
\hline 23 & B2 & 7.700 & 3.500 & 458 \\
\hline 24 & A2 & 7.500 & 1.695 & 311 \\
\hline 25 & $\mathrm{~A} 1$ & 7.410 & 1.920 & 770 \\
\hline 26 & $\mathrm{C} 2$ & 7.400 & 3.056 & 272 \\
\hline 27 & A1 & 7.340 & 2.800 & 865 \\
\hline 28 & A3 & 7.300 & 3.040 & 43 \\
\hline 29 & A1 & 7.200 & 2.685 & 508 \\
\hline 30 & A2 & 7.200 & 2.170 & 628 \\
\hline 31 & $\mathrm{~A} 1$ & 7.120 & 2.550 & 268 \\
\hline 32 & A3 & 7.090 & 2.100 & 157 \\
\hline 33 & $\mathrm{~A} 2$ & 7.010 & 3.600 & 282 \\
\hline 34 & A2 & 6.950 & 3.220 & 379 \\
\hline 35 & A3 & 6.840 & 3.660 & 429 \\
\hline 36 & B2 & 6.800 & 2.726 & 270 \\
\hline
\end{tabular}

\begin{tabular}{|c|c|c|c|c|}
\hline 37 & A2 & 6.620 & 2.010 & 403 \\
\hline 38 & A2 & 6.600 & 1.570 & 408 \\
\hline 39 & A1 & 6.520 & 2.320 & 654 \\
\hline 40 & A2 & 6.500 & 2.535 & 299 \\
\hline 41 & $\mathrm{C} 2$ & 6.310 & 2.030 & 433 \\
\hline 42 & B3 & 6.300 & 3.100 & 262 \\
\hline 43 & A1 & 6.210 & 1.910 & 829 \\
\hline 44 & $\mathrm{C} 1$ & 6.210 & 3.060 & 866 \\
\hline 45 & B2 & 6.150 & 1.190 & 255 \\
\hline 46 & A3 & 6.150 & 2.010 & 397 \\
\hline 47 & A1 & 6.150 & 2.540 & 794 \\
\hline 48 & $\mathrm{C} 1$ & 6.100 & 2.196 & 484 \\
\hline 49 & B2 & 6.100 & 2.100 & 446 \\
\hline 50 & $\mathrm{C} 2$ & 6.050 & 1.960 & 341 \\
\hline 51 & $\mathrm{C} 1$ & 6.040 & 4.010 & 854 \\
\hline 52 & A2 & 5.900 & 1.899 & 346 \\
\hline 53 & A2 & 5.900 & 2.065 & 278 \\
\hline 54 & C3 & 5.870 & 1.080 & 134 \\
\hline 55 & A2 & 5.800 & 2.300 & 637 \\
\hline 56 & A2 & 5.800 & 1.218 & 270 \\
\hline 57 & B2 & 5.800 & 2.940 & 210 \\
\hline 58 & $\mathrm{C} 2$ & 5.680 & 1.860 & 348 \\
\hline 59 & A3 & 5.660 & 1.300 & 167 \\
\hline 60 & A2 & 5.660 & 2.660 & 321 \\
\hline 61 & A2 & 5.570 & 2.140 & 269 \\
\hline 62 & $\mathrm{C} 3$ & 5.570 & 1.640 & 130 \\
\hline 63 & $\mathrm{C} 2$ & 5.560 & 2.940 & 313 \\
\hline 64 & A2 & 5.500 & 1.100 & 125 \\
\hline 65 & A2 & 5.446 & 2.490 & 261 \\
\hline 66 & A1 & 5.420 & 1.790 & 662 \\
\hline 67 & A2 & 5.330 & 1.790 & 294 \\
\hline 68 & A1 & 5.200 & 1.840 & 558 \\
\hline 69 & $\mathrm{C} 1$ & 5.200 & 1.700 & 569 \\
\hline 70 & $\mathrm{C} 2$ & 5.200 & 1.800 & 359 \\
\hline 71 & A1 & 5.180 & 1.180 & 168 \\
\hline 72 & $\mathrm{C} 2$ & 5.100 & 1.800 & 547 \\
\hline 73 & B3 & 5.070 & 2.560 & 182 \\
\hline 74 & A2 & 5.000 & 1.780 & 213 \\
\hline 75 & A1 & 4.840 & 1.910 & 218 \\
\hline 76 & A3 & 4.700 & 2.100 & 152 \\
\hline 77 & A3 & 4.600 & 1.340 & 172 \\
\hline 78 & $\mathrm{C} 1$ & 4.500 & 900 & 684 \\
\hline
\end{tabular}




\begin{tabular}{|c|c|c|c|c|}
\hline 79 & A3 & 4.480 & 1.350 & 125 \\
\hline 80 & A3 & 4.450 & 1.570 & 339 \\
\hline 81 & A1 & 4.400 & 1.425 & 285 \\
\hline 82 & A2 & 4.400 & 1.843 & 179 \\
\hline 83 & A1 & 4.400 & 1.600 & 782 \\
\hline 84 & A1 & 4.380 & 1.670 & 537 \\
\hline 85 & A2 & 4.310 & 1.220 & 233 \\
\hline 86 & A2 & 4.300 & 1.556 & 279 \\
\hline 87 & A1 & 4.200 & 1.667 & 65 \\
\hline 88 & A1 & 4.200 & 1.734 & 55 \\
\hline 89 & A2 & 4.170 & 1.750 & 420 \\
\hline 90 & $\mathrm{C} 2$ & 4.100 & 1.455 & 39 \\
\hline 91 & C3 & 4.090 & 610 & 36 \\
\hline 92 & A2 & 4.070 & 1.510 & 296 \\
\hline 93 & B3 & 4.000 & 1.430 & 30 \\
\hline 94 & B3 & 4.000 & 780 & 70 \\
\hline 95 & B3 & 3.900 & 600 & 4 \\
\hline 96 & A1 & 3.860 & 1.400 & 524 \\
\hline 97 & $\mathrm{C} 1$ & 3.800 & 1.774 & 406 \\
\hline 98 & A3 & 3.690 & 1.420 & 36 \\
\hline 99 & $\mathrm{C} 2$ & 3.600 & 950 & 28 \\
\hline 100 & A2 & 3.530 & 1.660 & 274 \\
\hline 101 & B3 & 3.530 & 1.460 & 171 \\
\hline 102 & A2 & 3.500 & 1.160 & 182 \\
\hline 103 & $\mathrm{C} 2$ & 3.500 & 1.529 & 252 \\
\hline 104 & A2 & 3.450 & 1.480 & 338 \\
\hline 105 & A2 & 3.400 & 1.580 & 439 \\
\hline 106 & A3 & 3.280 & 1.270 & 164 \\
\hline 107 & $\mathrm{C} 2$ & 3.010 & 1.570 & 109 \\
\hline 108 & B2 & 2.800 & 873 & 64 \\
\hline 109 & C3 & 2.600 & 553 & 24 \\
\hline 110 & C3 & 1.370 & 410 & 18 \\
\hline
\end{tabular}

\section{Resultados}

El número de leucocitos $/ \mathrm{ml}^{3}$ estuvo entre 13.180 y 1.370 , el número de linfocitos $/ \mathrm{ml}^{3}$ entre 4.010 y 410 , y el de $\mathrm{CD} 4 / \mu \mathrm{l}^{3}$ entre 1.039 y 4.

Se observó que los porcentajes de pertenencia al conjunto $\mathrm{A} \cup \mathrm{C}$ de acuerdo a cada rango se encontraron entre $80 \%$ y $100 \%$, mientras que para el conjunto $B \cup D$ estuvieron entre $56,25 \%$ y $100 \%$ y para la intersección $(A \cup C) \cap(B \cup D)$ variaron entre $50 \%$ y
$100 \%$. El porcentaje de pertenencia de la totalidad de casos al conjunto A $\cup \mathrm{C}$ fue de $92,73 \%$, mientras que para $\mathrm{B} \cup \mathrm{D}$ se encontró un porcentaje de pertenencia de $81,82 \%$. El porcentaje de pertenencia al conjunto compuesto por la intersección $(A \cup C) \cap(B \cup D)$ de la totalidad de casos fue de $77,27 \%$, ver tabla 2 , siendo superior al $80 \%$ en 5 de los 8 rangos establecidos.

Se observa además que la intersección $(A \cup C) \cap(B \cup D)$ presenta porcentajes de pertenencia que aumentan progresivamente desde el rango de 8.000 a 7.000 , de tal modo que los rangos inferiores a 5.000 y $4.000 / \mathrm{ml}^{3}$ predicen valores de menos de $570 \mathrm{CD} 4 /$ $\mu^{3}$ con un porcentaje de efectividad de $90 \%$ y $100 \%$ respectivamente. Aunque en este trabajo se obtiene un porcentaje de acierto relativamente alto en la predicción del rango de 5.999 a 5.000 (82,61\%), es necesario realizar nuevos estudios para establecer con mayor precisión la efectividad de la predicción para este rango.

Gracias a las predicciones obtenidas, el software desarrollado permite hallar el número de $\mathrm{CD} 4 / \mathrm{Ll}^{3}$ a partir de los valores de leucocitos y linfocitos del cuadro hemático convencional con base la teoría de conjuntos, junto con el porcentaje de efectividad de la predicción obtenida, siendo aplicable a la clínica para cada caso particular, independientemente de los valores de la carga viral, hemoglobina, edad, sexo o medicamentos.

\section{Discusión}

Este es el primer trabajo en el que se predice el conteo de $\mathrm{CD} 4 / \mathrm{\mu l}^{3}$, a partir del conteo de leucocitos y linfocitos, basado en la teoría de conjuntos, con un porcentaje superior al $80 \%$ para 5 de los 8 rangos medidos y específicamente, con valores de $90 \%$ y $100 \%$, para los valores inferiores a 5.000 y 4.000 leucocitos $/ \mathrm{ml}^{3}$ respectivamente, independientemente de las características del virus o de variables del paciente, logrando una abstracción simplificadora general y de aplicación clínica.

Para todos los rangos el porcentaje de pertenencia al conjunto $A \cup C$ es superior al porcentaje de pertenencia al conjunto $\mathrm{B} \cup \mathrm{D}$, a pesar de esto la observación del comportamiento de los porcentajes de pertenencia de los conjuntos de acuerdo a los rangos, con base en las leyes de la teoría de conjuntos permite una predicción con un porcentaje de efectividad que oscila 
Tabla 2. Número de elementos que conforman los conjuntos establecidos de acuerdo a cada rango y porcentaje de efectividad de la predicción de acuerdo con la teoría de conjuntos.

\begin{tabular}{|c|c|c|c|c|c|c|c|}
\hline \multirow{2}{*}{ LEUCOCITOS } & \multirow{2}{*}{$\begin{array}{l}\text { No. casos } \\
\text { por rango }\end{array}$} & \multicolumn{2}{|c|}{$A \cup C$} & \multicolumn{2}{|c|}{$B \cup D$} & \multicolumn{2}{|c|}{$(A \cup C) \cap(B \cup D)$} \\
\hline & & No. & $\%$ & No. & $\%$ & No. & $\%$ \\
\hline 10.000 O MAS & 6 & 6 & 100 & 5 & 83,33 & 5 & 83,33 \\
\hline 9.999 A 9.000 & 5 & 4 & 80 & 4 & 80 & 3 & 60 \\
\hline 8.999 A 8.000 & 6 & 6 & 100 & 5 & 83,33 & 5 & 83,33 \\
\hline 7.999 A 7.000 & 16 & 14 & 87,50 & 9 & 56,25 & 8 & 50 \\
\hline 6.999 A 6.000 & 18 & 15 & 83,33 & 12 & 66,67 & 11 & 61,11 \\
\hline 5.999 A 5.000 & 23 & 21 & 91,30 & 21 & 91,30 & 19 & 82,61 \\
\hline 4.999 A 4.000 & 20 & 20 & 100 & 18 & 90 & 18 & 90 \\
\hline 3.999 O MENOS & 16 & 16 & 100 & 16 & 100 & 16 & 100 \\
\hline TOTAL & 110 & 102 & 92,73 & 90 & 81,82 & 85 & 77,27 \\
\hline
\end{tabular}

entre el $82,61 \%$ y el $100 \%$ en los rangos inferiores a 6.000. Estos altos porcentajes encontrados en la asociación entre leucocitos y linfocitos, junto con la evaluación del comportamiento de los CD4 respecto a los diferentes rangos de leucocitos permite establecer una predicción de los rangos de valores de $\mathrm{CD} 4 / \mu \mathrm{l}^{3}$, con base en el valor de leucocitos/ml3, con predicciones superiores al $80 \%$ y que aumentan a un $90 \%$ en un rango de 5.000 a 4.000 leucocitos, y siendo totalmente efectiva, con un porcentaje del 100\% cuando se presenten menos de 4.000 leucocitos.

A pesar de los esfuerzos de la OMS para proporcionar terapia de combinación antirretroviral en países con pocos recursos $(9,10)$, y de la disminución de los costos del tratamiento, la citometría de flujo para el conteo de linfocitos T CD4, sigue siendo inaccesible en la mayoría de los países con pocos recursos (5). Dada la magnitud de la pandemia de VIH/SIDA a nivel mundial y especialmente en países en vías de desarrollo, resulta de gran importancia desarrollar métodos de bajo costo para el seguimiento del sistema inmune de los pacientes. El cuadro hemático es una prueba que por su bajo costo presenta una alta cobertura a nivel mundial, sin embargo, no permite establecer el número de $\mathrm{CD} 4 / \mathrm{ll}^{3}$, que es fundamental en la evaluación de esta patología y la toma de decisiones clínicas como la iniciación del tratamiento antirretroviral, o la vigilancia de la respuesta al tratamiento (5). La determinación en corto tiempo de los cambios en el conteo de CD4 podría ayudar a los médicos a prevenir las infecciones oportunistas (11) y servir como factor pronóstico de mortalidad en pacientes embarazadas (12), en países en vías de desarrollo. El conteo de linfocitos totales puede ser importante en decisiones del tratamiento para pacientes sintomáticos cuando no es posible tener el conteo de CD4 (13); de tal forma que es de gran utilidad el establecimiento de una medida predictiva del número de $\mathrm{CD} 4$ con base en el número de leucocitos y linfocitos totales, que permita además la disminución de costos para el seguimiento de pacientes con VIH/SIDA.

Algunos modelos de predicción de la tasa de disminución de linfocitos T CD4 han sido propuestos en aprendizaje de máquinas como el desarrollado por Singh y Mars (11) basado en la programación de máquinas con algoritmos específicos que se basan en el valor de la carga viral y el número de semanas respecto al inicio del conteo de CD4, estableciendo una predicción con una exactitud del $83 \%$ con respecto al valor real. En el contexto de la epidemiología, se han estudiado factores de predicción de linfocitos CD4; ejemplo de ello es un estudio de corte transversal que predijo recuentos de linfocitos CD4 <200 células/ $\mathrm{mm}^{3}$, a partir de los valores combinados de linfocitos $\mathrm{T}$ totales y hemoglobina, cuya sensibilidad y especificidad fue mayor al $80 \%$ en hombres, pero menor al $65 \%$ en mujeres (14). Otra metodología, plantea la predicción de la distribución de los recuentos de CD4 en pacientes seropositivos con base en patrones de variación de linfocitos $\mathrm{CD} 4$ de pacientes seronegativos y el tiempo de supervivencia después de la infección con VIH. Su aplicación predijo la variabilidad en la disminución del conteo de CD4 con una precisión del $76 \%$ y $82 \%$ en las poblaciones de Zambia y Sudáfrica 
(15), y $87 \%$ en datos de un trabajo posterior (16). Sin embargo la gran variabilidad en la tasa de disminución de esta población celular de persona a persona, establece el reto de entender la variación de los niveles de células CD4 en poblaciones e individuos específicos (15). En contraposición, en este trabajo es posible establecer relaciones matemáticas generales, que son aplicables a cada caso particular independientemente de consideraciones de carácter estadístico o epidemiológico, dándole importancia a la comprensión objetiva de la interacción del sistema inmune con el VIH. Con esta perspectiva Vélez y Torrealea desarrollaron desde herramientas informáticas un modelo descriptivo de la dinámica de la respuesta inmune ante el VIH, pero no plantea predicciones, como el conteo de CD4 (17).

Otras metodologías se han enfocado en evaluar la respuesta del virus a medicamentos determinados, entre ellas, las que se basan en aprendizaje de máquinas con redes neurales para predecir la carga viral de pacientes con HIV a partir del genotipo y datos del tratamiento, realizando algoritmos con una precisión del 75\% (18), y $69 \%$ (19). También se han desarrollado algoritmos para predecir el éxito o fracaso de tratamientos, que tienen en cuenta la respuesta dicotómica del virus (20) obteniendo una precisión del $80 \%$, o basados en el grado de resistencia del virus a medicamentos, de acuerdo a sus características genotípicas (21). En este trabajo se estudió el fenómeno partiendo de siete casos, siendo observados desde la teoría de conjuntos, y mediante una inducción se definen los conjuntos específicos para las predicciones, que son luego corroboradas en la experiencia al tomar los 103 casos restantes.

La teoría de conjuntos ha sido de utilidad para el estudio de otros fenómenos en inmunología. Por ejemplo, a partir de la teoría de conjuntos se caracterizó la unión de péptidos al HLA de clase II, hallando un comportamiento determinable a partir de reglas encontradas experimentalmente en péptidos nonámeros específicos, logrando diferenciar péptidos de unión y no unión (22). También con la teoría de conjuntos (23) se caracterizó el fenómeno de alta unión de la proteína de membrana MSP-1 al receptor de glóbulo rojo, acertando en $100 \%$ de los casos estudiados y encontrando órdenes matemáticos que diferencian de forma objetiva y reproducible los péptidos que presentan alta unión, de los que no. Estos fenómenos, del mismo modo que la variación de las poblaciones de leucocitos $y$ linfocitos en pacientes con $\mathrm{VIH}$, han sido estudiados tradicionalmente desde un pensamiento empírico, no abstracto ni universalizador. Sin embargo este trabajo, así como los trabajos mencionados, evidencian la utilidad de la comprensión de los fenómenos de la inmunología desde teorías físicas y matemáticas, en este caso la teoría de conjuntos, y sus implicaciones a nivel experimental y clínico.

En otras áreas de la medicina, la aplicación de teorías físicas y matemáticas también ha permitido desarrollar resultados aplicables en la práctica, no sólo a nivel clínico y experimental sino en el campo de la salud pública. Por ejemplo, se desarrolló un método de diagnóstico clínico del Holter a partir de los sistemas dinámicos y proporciones de la entropía de los atractores, que permite diferenciar normalidad, enfermedad crónica y enfermedad aguda, así como la evolución entre estados (24). También fue realizada una generalización teórica fractal de la totalidad de arterias coronarias en el proceso de estenosis y reestenosis con base en el concepto de Armonía Matemática Intrínseca (25). Se cuantificó la dinámica semanal de malaria en todos los municipios de Colombia y teniendo en cuenta la relación entropía/constante de Boltzmann predijo la aparición de brotes con una efectividad del 99,86\% (26). De igual forma se desarrollaron teorías predictivas para el fenómeno de unión de péptidos al HLA clase II, y para la unión de péptidos de proteínas de malaria al glóbulo rojo, que al ser comparados con los resultados experimentales presentaron un porcentaje de efectividad del 100\% en el primer caso, mientras que en el segundo se establecieron valores de sensibilidad y una especificidad del $95 \%$ y del $90 \%$, respectivamente $(7,27)$. Los resultados obtenidos revelan que fenómenos en diferentes campos de la medicina obedecen a órdenes físico-matemáticos si son conceptualizados desde abstracciones teóricas, o sea desde leyes y teorías físicas y matemáticas, logrando aplicaciones clínicas independientemente de consideraciones causales.

\section{Dedicación}

A nuestros hijos y a las personas infectadas con VIH para quienes trabajamos.

\section{Agradecimientos}

A la Clínica del Country, en especial al Dr. Andrés Rodríguez, a la Dra. Natalia Malaver, al Dr. Tito Tulio Roa y a todas las personas que trabajan en el Centro de Investigaciones por su apoyo decidido para que se concreten estos resultados. 
A la Universidad Militar Nueva Granada, especialmente a Vicerrectoría de Investigaciones, y a la Facultad de Medicina por apoyar nuestras investigaciones. Este artículo es resultado del proyecto MED-736 financiado por el Fondo de Investigaciones de la Universidad Militar Nueva Granada, por lo cual agradecemos su apoyo.

A Germán Puerta, interno especial de Física y Matemáticas aplicadas a la Medicina de la Universidad Militar Nueva Granada en la Clínica del Country, al Dr. Juan Bravo, profesor de la Línea de profundización en Infectología de la Facultad de Medicina de la Universidad Militar Nueva Granada, a Natalia Perdomo y Álvaro Faccini, internos especiales en Infectología de la Universidad Militar Nueva Granada, por su colaboración eficiente y oportuna.

\section{Referencias}

1. WHO, UNAIDS. Joint United Nations Programme on HIV/ AIDS. UNAIDS Report on the Global AIDS epidemic 2010. p. 18 [Internet] [Actualizado 2010]; Citado 2011 Julio 12]. Disponible en: http://www.UNAIDS.org

2. Sabogal A, Grupo ITS, Subdirección de vigilancia y control en salud pública. Informe de VIH-SIDA Colombia periodo XIII año 2009.

3. Instituto nacional de salud, Colombia. Subdirección de vigilancia y control en salud pública. Boletín epidemiológico semanal, semana epidemiológica numero 49 de 2010 (5 al 11 de diciembre de 2010). Hepatitis B. p. 15.

4. Abbas A, Lichtman A, Pober J. Inmunología celular y molecular 2 a ed. Madrid: McGraw Hill; 1995.

5. Zijenah, L, Kadzirange G, Madzime S, Borok M, Mudiwa Ch, Tobaiwa $\mathrm{O}$, et al, Affordable flow cytometry for enumeration of absolute CD4+ T-lymphocytes to identify subtype C HIV1 infected adults requiring antiretroviral therapy (ART) and monitoring response to ART in a resource-limited setting. Journal of Translational Medicine. 2006; 4:33.

6. VIH y SIDA; Los virus del Sida. [Internet] [Actualizado 2000; Consultado 2011 Mar 9] Disponible en: http://www.ctv.es/ USERS/fpardo/virus.htm.

7. Rodríguez J. Teoría de unión al HLA clase II teorías de Probabilidad Combinatoria y Entropía aplicadas a secuencias peptídicas. Inmunología. 2008; 27(4):151-166.

8. Rodríguez J. Comportamiento fractal del repertorio T específico contra el alérgeno Poa P9. Rev Fac Med Univ Nac Colomb. 2005; 53(2):72-78.

9. WHO, UNAIDS. Joint United Nations Programme on HIV/ AIDS. Progress on global access to antiretroviral therapy: an update on " 3 by 5 ". Vol. 2005.

10. WHO, UNAIDS. Joint United Nations Programme on HIV/ AIDS AIDS. Epidemic update 2005.

11. Singh Y, Mars M. Support vector machines to forecast changes in CD4 count of HIV-1 positive patients. Scientific Research and Essays. 2010; 5(17):2384-2390.

12. Brown E, Otieno P, Mbori-Ngacha D, Farquhar C, Obimbo E, Nduati R, et al. Comparison of CD4 Cell Count, Viral Load, and Other Markers for the Prediction of Mortality among HIV-1-Infected. J Infect Dis. 2009; 199(9): 1292-1300.
13. Daka D, Loha E. Relationship between Total Lymphocyte count (TLC) and CD4 count among peoples living with HIV, Southern Ethiopia: a retrospective evaluation. AIDS Res Ther. 2008; 5:26.

14. Budiono W. Total lymphocyte count and hemoglobin combined to predict CD4 lymphocyte counts of less than 200 cells/ mm(3) in HIV/AIDS. Acta Med Indones. 2008; 40(2):59-62.

15. Williams B, Korenromp E, Gouws E, Schmid G, Auvert B, Dye C. HIV Infection, Antiretroviral Therapy, and CD4+ Cell Count Distributions in African Populations. Journal of Infectious Diseases. 2006;194:1450-1458.

16. Williams BG, Korenromp EL, Gouws E and Dye C. The rate of decline of CD4 T-cells in people infected with HIV. Cornell university library 2009 archive in arXiv:0908.1556v1.

17. Vélez N, Torrealdea J. Modelado en dinámica de sistemas de la respuesta inmune ante la infección del VIH-1. Ini Inv. 2006; 1-11.

18. Wang D, DeGruttola V, Hammer S, Harrigan R, Larder B, Wegner S, et al. A Collaborative HIV Resistance Response Database Initiative: Predicting Virological Response Using Neural Network Models. Poster presentation at: The XI International HIV Drug Resistance Workshop: Seville, 2002.

19. Larder B, Wang D, Revell A, Montaner J, Harrigan R, De Wolf $\mathrm{F}$, et al. The development of artificial neural networks to predict virological response to combination HIV therapy. Antivir Ther. 2007; 12(1):15-24.

20. Altmann A, Rosen-Zvi M, Prosperi M, Aharoni E, Neuvirth N, Schülter E, et al. Comparison of Classifier Fusion Methods for Predicting Response to Anti HIV-1 Therapy. PLoS ONE 2008; 3(10):e3470

21. Altman A, Däumer M, Beerenwinkel N, Peres Y, Schülter $\mathrm{E}$, Büch J, et al. Predicting the Response to Combination Antiretroviral Therapy: Retrospective Validation of geno2pheno-THEO on a Large Clinical Database, JID. 2009; 199:999-1006.

22. Rodríguez J. Teoría de conjuntos aplicada a la caracterización matemática de unión de péptidos al HLA clase II. Rev Cienc Salud. 2008; 6(1):9-15

23. Rodríguez J. Diferenciación matemática de péptidos de alta unión de MSP-1 mediante la aplicación de la teoría de conjuntos. Inmunología. 2008; 27(2):63-68.

24. Rodríguez J. Entropía proporcional de los sistemas dinámicos cardiacos. Predicciones físicas y matemáticas de la dinámica cardiaca de aplicación clínica. Rev Col Cardiol. 2010; 17(3):115-129.

25. Rodríguez J, Prieto S, Correa C, Bernal P, Puerta G, Vitery S, et al. Theoretical generalization of normal and sick coronary arteries with fractal dimensions and the arterial intrinsic mathematical harmony. BMC Med Phys 2010; 10:1-6.

26. Rodríguez J. Método para la predicción de la dinámica temporal de la malaria en los municipios de Colombia. Rev Panam Salud Pública. 2010; 27(3):211-218.

27. Rodríguez J, Bernal P, Prieto S, Correa C. Teoría de péptidos de alta unión de malaria al glóbulo rojo. Predicciones teóricas de nuevos péptidos de unión y mutaciones teóricas predictivas de aminoácidos críticos. Inmunología. 2010; 29(1):7-19. 